\title{
Virtual Machine Migration in Cloud Computing
}

\author{
Pankajdeep Kaur and Anita Rani \\ CSE Department \\ Gndu, Rc, Jalandhar \\ pankajdeepkaur@gmail.com \\ anitasaroay@yahoo.in
}

\begin{abstract}
Cloud computing is the delivers the computing services over the internet. Cloud services help individuals and organization to use data that are managed by third parties or another person at remote locations. Virtual Machine (VM) is an emulation of a particular computer system. In cloud computing, Virtual machine migration is a useful tool for migrating Operating System instances across multiple physical machines. It is used to load balancing, fault management, low-level system maintenance and reduce energy consumption. There are various techniques and parameters available for VM migration. This paper presents the various virtual machine migration techniques.
\end{abstract}

Keywords: VM Live migration, Post-copy, Network aware Migration, Pre-copy Techniques

\section{Introduction}

Cloud computing distributes the computing tasks to the resource pool made from a large number of computers.

VMs refer to one instance of an operating system along with one or more applications running in an isolated partition within the computer. There will be multiple virtual machines running on top of a single physical machine. When one physical host gets overloaded, it may be required to dynamically transfer certain amount of its load to another machine with minimal interruption to the users. This process of moving a virtual machine from one physical host to another is termed as migration. In the past, to move a VM between two physical hosts, it was necessary to shut down the VM, allocate the needed resources to the new physical host, move the VM files and start the VM in the new host. Live migration makes possible for VMs to be migrated without considerable downtime. The transfer of a VM actually refers to the transfer of its state. This includes its memory, internal state of the devices and that of the virtual CPU. Among these, the most

Time-consuming one is the memory transfer. Two parameters are considered while performing the live VM-migration are:

1) Downtime- Down time refers to the time during which the service of the VM is not available.

2) Migration Time- Migration time refers to the total amount of time required to transfer a virtual machine at source to destination node without affecting its availability.

Virtualization is major concept of cloud computing. It is becoming popular in cloud computing environments due to the advantage of server consolidation, resource isolation and live migration.

\section{Related Work}

Virtualization help in partitioning of one physical machine into number of virtual machines that runs concurrently and it also shares the same physical resources. Virtual 
machine migration is done from one physical machine to another machine. It is used for load balancing and physical machine fault tolerant. It can also be used to reduce power consumption in cloud data centers.

Virtual Machine Migration methods are divided into two types: 1) Hot (live) migration- Virtual machine keeps running while migrating and does not lose its status.

2) Cold (non-live) migration- The status of the VM loses and user can notice the service interruption.

User doesn't feel any interruption in service in hot (live) migration. In live migration process, the state of a virtual machine to migrate is transferred. The state consists of its memory contents and local file system. Local file system need not be transferred. In cold migration, first, VM is suspended, then its state is transferred, at last VM is resumed at destination host.

a) Live VM Migration- Live migration [8] is a technology used for load balancing and optimization of VM deployment in data centers. With the help of live migration, VMs can be transferred to another node without shutting down. Live migration is classified into two steps -

(i) Control is switched to the destination.

(ii) Data Transferring (memory/disk) to the destination.

Pre-copy- In this, first Memory is transferred and after this execution is transferred. The pre-copy method is used to transfer the memory to the destination node over a number of iterations.

Post-copy-In this, First execution is transferred and after this, memory is transferred. Unlike pre-copy, in post copy the Virtual CPU and devices on the destination node is transfer in the first step and starts the execution in second step. Following metrics are used to measure the performance of live migration.

i) Preparation- In this, resources are reserved on the destination which performed various operations.

ii) Downtime- Time during which the VM on the source host is suspended

iii) Resume- It does the instantiation of VM on the destination but with the same state as suspended source.

iv) Total time- The total time taken in completion of all these phases is called Total Migration time.

b) Live VM Migration Techniques- The pre copy [2] migration iteratively copies the memory pages from the source machine to the destination host, without ever stopping the execution of the VM. The iterative nature of the algorithm is due to the dirty pages, i.e. memory pages that have been modified in the source host since last page transfer must be sent again to the destination host. If the rate of updating of pages is very high, migration time will rise to a very high value. But the advantage of this approach is that all updating are available at the destination host. It can be activated any time. Every VM will have some set of pages that it updates very frequently and which are therefore poor candidates for pre-copy migration. 


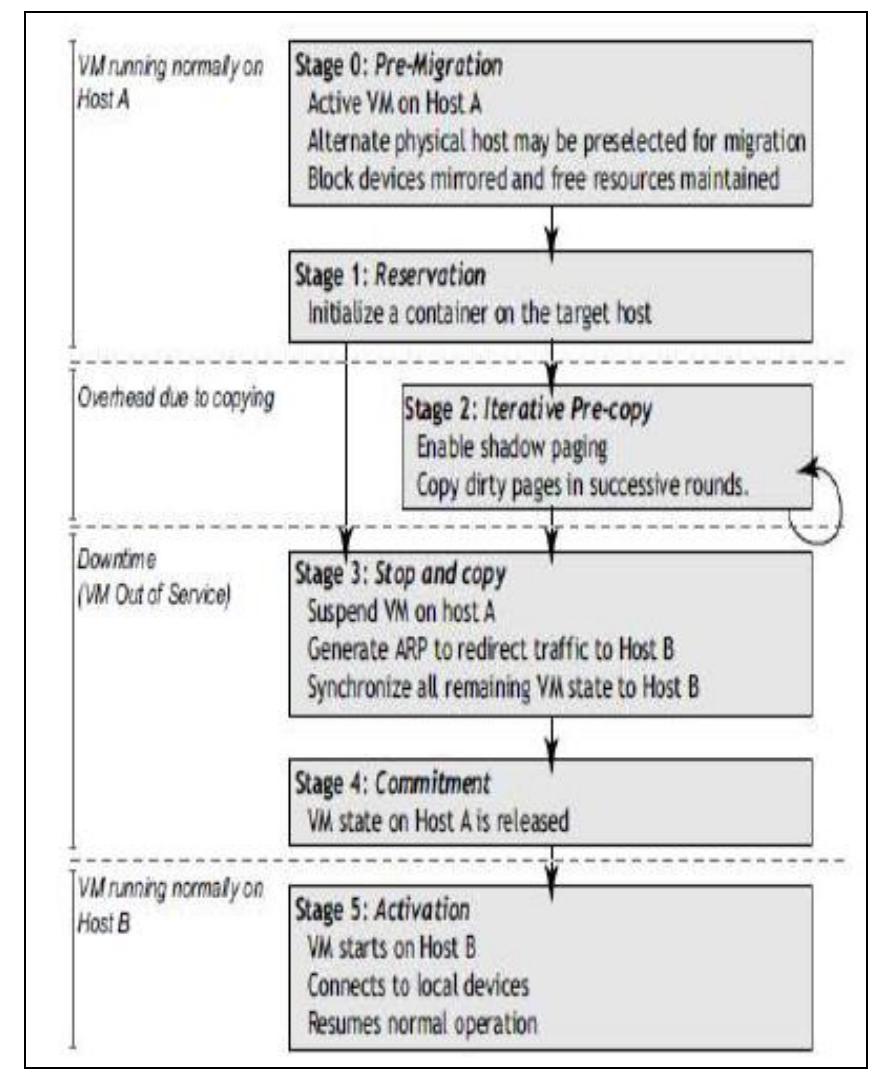

Figure 1. Pre-Copy Algorithm [2]

There are 3 phases in live migration approach:

Warm-up phase-In this, copies all the memory pages from source to destination while the VM is still running on the source. If some memory pages change during memory copy process dirty pages, they will be re-copied until the rate of recopy pages is not less than dirty page rate.

Stop and Copy phase- In this, the VM will be stopped in source and the remaining dirty pages will be copied to the destination and VM will be resumed in destination.

Pre-Copy Phase- At this stage, the VM continuously run, and its memory is copied page wise from the source to the target host. It starts with transferring all active memory pages.
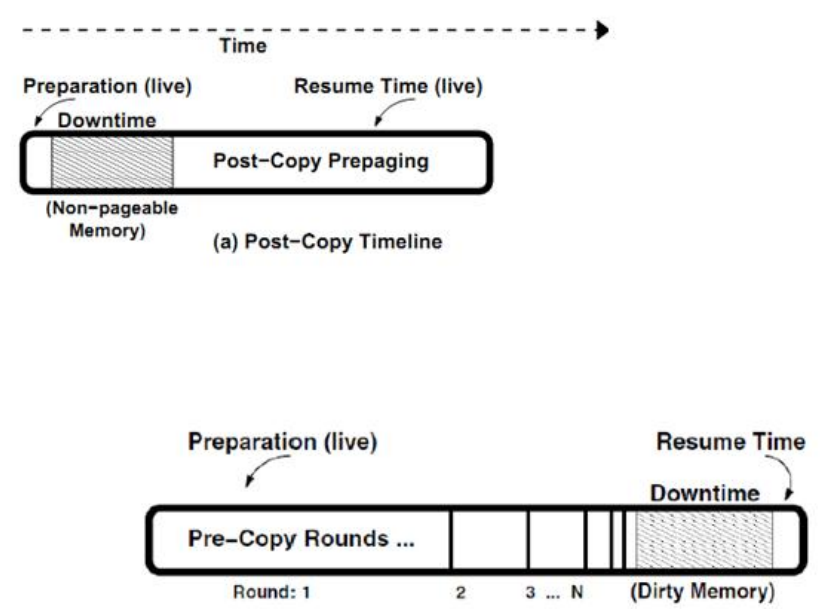

Figure 2. Timeline for Post-copy and Pre-copy [3] 
In modified pre-copy approach [3], a framework is constructed that includes preprocessing phase in traditional pre-copy based live migration so that the amount of transferred data is reduced. For preprocessing, they proposed the working set prediction algorithm. Applying the proposed algorithm which combined LRU (Least Recently Used) cache with splay tree algorithm, the system can reduce the amount transferred memory page. In an approach to optimize live virtual machine migration based on pre-copy algorithm, memory compression is used.

The basic idea of live migration algorithm was first proposed in [4]. First Hypervisor marks all pages as dirty, then algorithm iteratively transfer dirty pages across the network until the number of pages remaining to be transferred is below a certain threshold or a maximum number of iterations is reached. Then Hypervisor mark transferred pages as clean, Since VM operates during live migration, so already transferred memory pages may be dirtied during iteration and must need to be re-transferred. The VM is suspended at some point on the source for stopping further memory writes and transfer remaining pages. After transferring all the memory contents, the $\mathrm{VM}$ resumes at destination server.

In [5] describes the design and implementation of a system that uses virtual machine technology to provide fast, transparent application migration; neither the applications nor the operating systems need to be modified. Performance is measured with hundred virtual machines, migrating concurrently with standard industry benchmarks. It shows that for a variety of workloads, application downtime due to migration is less than a second.

A high performance VM migration design based on Remote Direct Memory Access was proposed in [6]. InfiniBand is an emerging interconnects providing feature such as Operating System bypass and RDMA. Remote Direct Memory Access is a direct memory access from the memory of one computer to that of another computer without involving their operating system. With the help of Remote Direct Memory Access remote memory can be read and write directly.

In [7] introduced a whole-system live migration technique, which transfers the whole system run time state, including CPU state, local disk storage and memory data of the virtual machine. They proposed a three phase migration algorithm as well as an incremental migration algorithm, which migrate the virtual machine back to the source machine in a very short total migration time.

In [8] presented a system for supporting the transparent, live wide-area migration of virtual machines which used local storage for their persistent state. This approach is transparent to the migrated VM, and does not interrupt open network connections to and from the VM during wide area migration, This ensure consistency of the VM's local persistent state at the source and the destination after migration and is able to handle highly write intensive workloads.

[9] Presented a technique to improve the performance of live migration. In this an optimized iterative pre-copy algorithm is used which reduce the dirty rate of VM.

In [10] presents, hybrid approach is used to takes the best of methods of live migration - pre and post-copy, in this, with processor state, they add a lot of useful information of state. This includes devices and frequently accessed pages of the VM, i.e. working set

In [11] Network aware VM Migration strategy is proposed. In this, migration of VM is purely base on network traffic and network latency and various strategies are used for migration. 

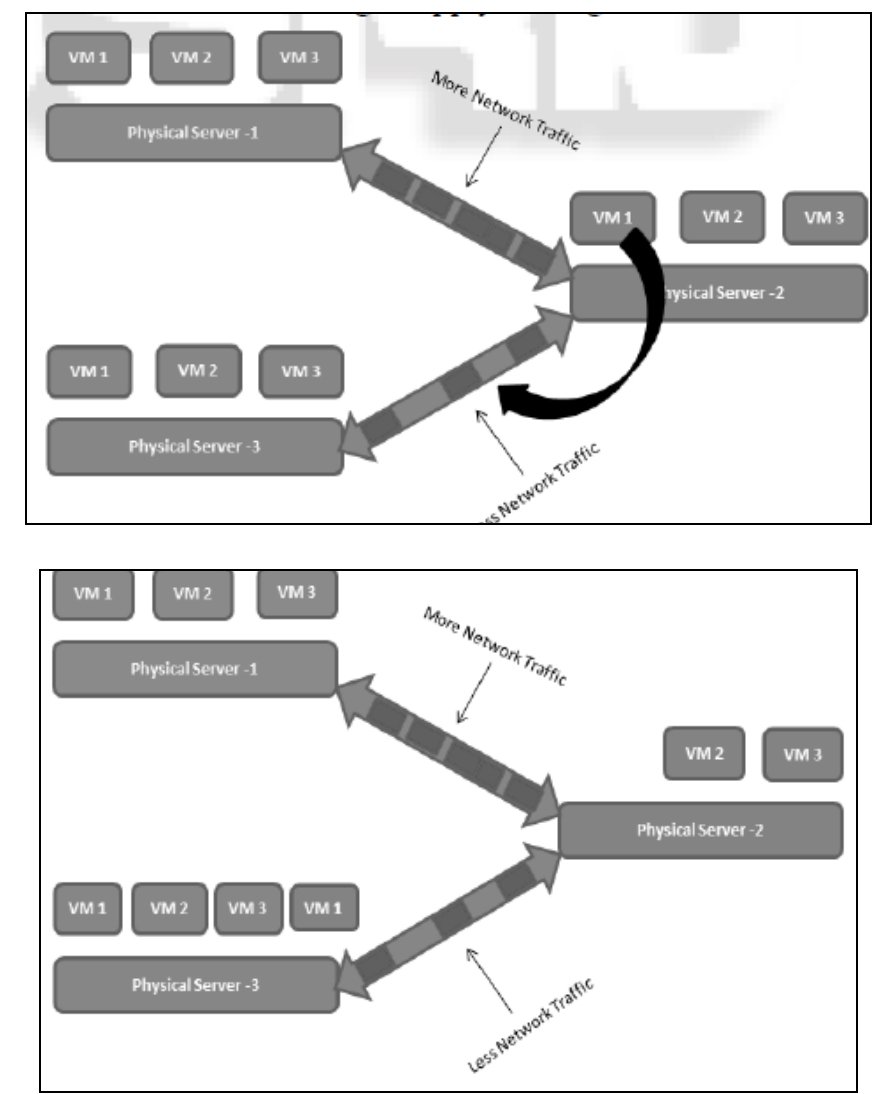

\section{Figure 3. Network Aware Migration (Before \& after migration [11]}

In [12] present an approach, VM placement and migration for data intensive application, which helps to minimize data transfer time. The proposed approach places the VMs on target physical machines and consider of the network conditions between the physical machines and the data storage.

b) Advantages: Various advantages of VM Migration are:

Load balancing: This reduces the inequality of resource usage levels across all the PMs in the cluster. This prevents some machines from getting overloaded in the presence of lightly loaded machines with sufficient spare capacity. Live migration can be used to balance the system. The overall system load can be balanced by migrating VMs from overloaded PMs to under-loaded PMs.

Server Consolidation: In order to reduce server sprawl in data centers, server consolidation algorithms are required. These algorithms are VM packing heuristics which try to pack as many VMs as possible on a PM so that resource usage is improved and unused or under-utilized machines can be turned off. Consolidation will result in reduced power consumption and thus reducing overall operational costs for data centre administrators.

Hotspot \& Coldspot Migration: The detection of hotspots and coldspot are always based on thresholds which are set by the data center owner or based on the Service Level Agreements specified by the clients. Usually, a higher resource usage value close to maximum is set as the upper threshold and a very low resource usage value is set as the lower threshold. PMs having resource usage values beyond the upper threshold are said to have formed hotspots, and whose usage values below the lower threshold are said to have formed coldspot. The former implies over-utilization and the latter implies underutilization, applicable across any resource dimension. 


\section{Future Scope}

VM Migration SLA Based- There is a limitation of vm migration technology, when it is used for SLA (Service level Agreements) the optimized data access might still go beyond the time requirement.

High Dirty Rate Memory Page -The problems like more page fault and higher total migration time occur when high dirty rate of memory pages even memory threshold technique applied.

\section{Conclusion}

This paper presents techniques of live migration of virtual machine. Live migration includes transfer of a running virtual machine over physical hosts. There are many techniques which are used to minimize the down time \& total migration time to provide better performance in low bandwidth. There is less number of network aware migration techniques available which helps more. With the increase in the popularity of cloud computing systems, virtual machine migrations across data centers and resource pools will be greatly beneficial to data center administrators. Live virtual machine migration is an indispensable tool for dynamic resource management in modern day data centers. In this paper presents the techniques of live virtual machine migration which takes considerable amount of migration time and downtime. Live migration of VMs should be designed in such a way so that downtime and migration time will be reduced.

\section{References}

[1] A. Aggarwal, "Comparative Review of Scheduling and Migration Approaches in Cloud Computing Environment”, IJEBEA International Association of Scientific Innovation and Research (IASIR), (2014)

[2] S. Mohan, "Survey on Live VM Migration Techniques", International Journal of Advanced Research in Computer Engineering \& Technology (IJARCET).

[3] M. Chawda and O. Kale, "Virtual Machine Migration Techniques in Cloud Environment, A Survey", International Journal for Scientific Research \& Development (IJSRD).

[4] Y. Kejiang, "Live migration of multiple virtual machines with resources", IEEE, (2014).

[5] K. Sato, H. Sato and S. Matsuoka, "A model-based algorithm for optimizing I/O intensive applications in clouds using vm-based migration", IEEE, (2014).

[6] T. Alexander, T. Setzer, "Network-aware migration control and scheduling of differentiated virtual machine workloads," IEEE Computer Society, (2013).

[7] H. Chen, H. Kang, G. Jiang and Y. Z. Stage, "Network-aware migration control and scheduling of differentiated virtual machine workloads", IEEE Computer Society, (2012).

[8] B. Hu, Z. Lei, Y. Lei, D. Xu and J. Li, "A time-series based pre copy approach for live migration of virtual machines", International Conference on Parallel and Distributed Systems (ICPADS).

[9] C. Xianqin and G. Xiaopeng, "Application-Transparent Live Migration for Virtual Machine on Network Security Enhanced Hypervisor", China communications, (2012).

[10] S. Akoush and R. Sohan, "Predicting the Performance of Virtual Machine Migration", IEEE International Symposium on Modeling, Analysis and Simulation of Computer and Telecommunication systems (MASCOTS'10), (2011).

[11] E. Zaw and N, Thein, "Improved Live VM Migration using LRU", International Journal of Computer Science and Telecommunications, (2011). March.

[12] H. Jin and L. Deng and S. Wu, "Live Virtual Machine Migration with Adaptive Memory Compression", (2010). 